Check for updates

Cite this: RSC Adv., 2019, 9, 35998

\title{
Inkjet assisted fabrication of planar biocompatible memristors
}

\author{
Georgii A. Illarionov, (D) a Denis S. Kolchanov, (D) a Oleg A. Kuchur, (D) ${ }^{a}$ \\ Mikhail V. Zhukov, (D) ab Ekaterina Sergeeva, (D) a Vladimir V. Krishtop, (D) ac \\ Alexandr V. Vinogradov (D) and Maxim I. Morozov (D) *a
}

\begin{abstract}
In this study we address a novel design of a planar memristor and investigate its biocompatibility. An experimental prototype of the proposed memristor assembly has been manufactured using a hybrid nanofabrication method, combining sputtering of electrodes, patterning the insulating trenches, and filling them with a memristive substance. To pattern the insulating trenches, we have examined two nanofabrication techniques employing either a focused ion beam or a cantilever tip of an atomic force microscope. Inkjet printing has been used to fill the trenches with the functional titania ink. The experimental prototypes have qualitatively demonstrated memristive current-voltage behavior, as well as high biocompatibility.
\end{abstract}

Received 7th October 2019

Accepted 25th October 2019

DOI: 10.1039/c9ra08114c

rsc.li/rsc-advances

artificial neural networks. This interest arises from two general research trends: adapting digital computational processes to native information processing mechanisms ${ }^{20,21}$ and investigation of the functional reserves of the biological neural networks. ${ }^{22,23}$ From the technological point of view, the electronic resistive switching memory is the common feature belonging to both biological and artificial electrical neurons. As such, it is considered as the frontier of feasible bionanointerfaces. The state of the art and future expectations in this field have recently been outlined in a topical review. ${ }^{24}$

Nowadays, in vitro studies of electrical activity in neuronal and other biological cells require special microelectrode arrays $^{25}$ and thus external wiring is needed to establish connection with a control unit or a memristive device. ${ }^{26}$ Alternatively, microelectrodes and memristive elements can be integrated into one device. In the most common topological arrangement, memristors are fabricated as a dielectric thin film sandwiched between the transverse arrays of top and bottom electrodes (so-called "crossbar latch"). This configuration is a key architecture proposed for integrated nanoscale electronics. ${ }^{27}$ Integration of crossbar memristors with the outer microelectrode array for in vitro experiments with biological cells or tissues would require an additional insulating layer and conductive channels between the inner and outer electrodes. This can be fabricated using conventional lithography. Alternatively, a layout with planar topology can be designed. Recently, several studies addressed the planar configuration of memristive elements. ${ }^{28-30}$ These elements were technologically performed using photolithography, leaving gaps between the pairs of metallic electrodes and filling them with a memristive composite. The gaps widths were reported to range from 10 $\mu \mathrm{m}{ }^{28,29}$ to $50-200 \mu \mathrm{m} .^{30}$ To achieve the memristive effect

${ }^{a}$ Laboratory of Solution Chemistry of Advanced Materials and Technologies, ITMO University, Lomonosova str. 9, St. Petersburg, 191002, Russia.E-mail: maximm@ alumni.ntnu.no; morozov@scamt-itmo.ru

${ }^{b}$ Institute for Analytical Instrumentation RAS, Ivan Chernykh str. 31-33, St. Petersburg, 198095, Russia

${ }^{c}$ Research Center, Ivanovo State Medical Academy, Sheremetevsky ave. 8, Ivanovo, 153012, Russia 
between so far distant electrodes, the authors used dielectric nanocomposite materials with silver nanoparticles. The use of silver nanoclusters allows for memristive effect based on reversible generation of conductive filaments through the electrically induced diffusive mechanism. ${ }^{31}$ However, the use of silver nanoparticles in biocompatible devices is risky, as their reported cytotoxicity at the cell level may be an issue. ${ }^{32}$ In this regard, an interesting solution would be the use of other than silver-based compounds with metal nanoclusters capable for realizing the same type of diffusive mechanism of memristivity. Recently, a promising effect of electroforming in gold nanoclusters has been demonstrated on a planar memristor prototype $(3 \mathrm{~mm} \times 3 \mathrm{~mm}$ ) fabricated by supersonic cluster beam deposition. ${ }^{33}$

The micrometer-scale resolution for the planar patterning of the diffusive-type memristors can be achieved by the conventional mask lithography. The use of mask lithography is however limited for in situ biological studies, so all the operations are allowed only prior to deposition of biological cells.

An alternative choice of planar memristive elements is represented by various metal oxides, whose memristance is based on the ionic drift mechanism. ${ }^{\mathbf{4 3 4 - 3 6}}$ However, their realization requires much thinner dielectric layer, as the theoretical memristance of the ionic drift system is shown to be inverse square dependent on the distance between the electrodes. ${ }^{4}$ In this case, however, the use of conventional mask lithography meets aberration challenges and thus becomes costly.

In this study, we propose a hybrid nanofabrication method for planar memristors, comprising the nanoscale patterning of insulating trenches by atomic force microscopy (AFM) and inkjet printing of the functional ink. This combination allows accurate manipulations at ambient environment and as such is prospective for designing, engineering, and prototyping electrical circuits with biological cells or tissues, both prior to and after their deposition. A prototype device with a series of memristor elements has been manufactured accordingly and thoroughly investigated. In order to investigate the biocompatibility of the materials used in the prototype, an assay for their biocompatibility has been performed using both neuronal (IMR-32, neuroblastoma) and non-neuronal (PHF, postnatal human fibroblasts) cell lines. Next, we report the main processing features for the elaborated prototype, the functional behaviour, and discuss the applicability, challenges, and further prospects of the proposed fabrication technique.

\section{Materials and methods}

\section{Device outline}

The concept of the proposed planar memristor element is as follows: a thin layer of metallic electrode is deposited on a glass substrate. The shape of the electrode has a narrow bottleneck area in the middle, where a thin trench across the electrode is formed. The depth of the trench overcomes the electrode thickness, so that the resulting narrow gap splits the original electrode into two terminals. Then the gap is filled by a memristive dielectric substance.
In our prototype samples, five memristor elements with planar $\mathrm{Au}-\mathrm{TiO}_{2}-\mathrm{Au}$ sandwiches were fabricated on the surface of glass substrates (size $76 \mathrm{~mm} \times 25 \mathrm{~mm} \times 1 \mathrm{~mm}$, roughness $<0.15 \mu \mathrm{m}$, Levenhuk, Russia). The electrodes were deposited by gold sputtering, while their shapes were profiled by photolithography. The electrode separating trenches were made by scratching with a cantilever tip of an atomic force microscope (AFM). In addition, a reference series of memristive elements were prepared with trenches made by focused ion beam (FIB) milling. The resulting gaps between the electrodes were filled with titanium dioxide ink, using a laboratory scale inkjet printer. The AFM-based fabrication process is schematically shown in Fig. 1. In the following subsections, the main processing steps will be described in detail.

Electrode fabrication. The glass substrate was washed with a detergent, deionized water, and acetone, and then dried in oven at $110{ }^{\circ} \mathrm{C}$ for $30 \mathrm{~min}$. A sequence of $3 \mathrm{~nm}$ chromium and $30 \mathrm{~nm}$ gold layers were sputtered on the substrate. The chromium intermediate layer was used for improving adhesion between gold and glass. The shapes of the electrodes were formed using photolithography. A positive photoresist (AZ 1505) was spin-coated on the gold surface. The surface patterning was performed using G-line lithography with a wavelength of $436 \mathrm{~nm}$. The unprotected part of the gold layer was removed by wet etching in a $1 \mathrm{wt} \%$ aqueous KCN solution for $20 \mathrm{~s}$ at $25{ }^{\circ} \mathrm{C}$. The remaining chromium layer was etched in a $\mathrm{Cr}$ etchant (CR-7) for $10 \mathrm{~s}$ at $25^{\circ} \mathrm{C}$. The protecting layer of the photoresist was removed in a bath with photoresist remover (AZ400T) taken at $50{ }^{\circ} \mathrm{C}$ for $1 \mathrm{~h}$. The result is shown in Fig. 2.

Trench forming. The electrode separating gaps were formed as thin trenches crossing the gold layers at their narrowest "bottleneck" areas.

The trenches of the first type were made mechanically, by scratching the gold surface with a cantilever tip of a scanning probe microscope (SPM) Ntegra Aura (NT-MDT, Russia), operating in the AFM contact mode. The cantilever was chosen with a hardness of $22 \mathrm{~N} \mathrm{~m}^{-1}$ (NT-MDT, Russia) and a tip curvature radius of $10 \mathrm{~nm}$. The scratching force was estimated as:

$$
F=\Delta Z k
$$

where $\Delta Z$ is the deflection of the probe between two fixed points, and $k$ is the stiffness of the cantilever. A calibration

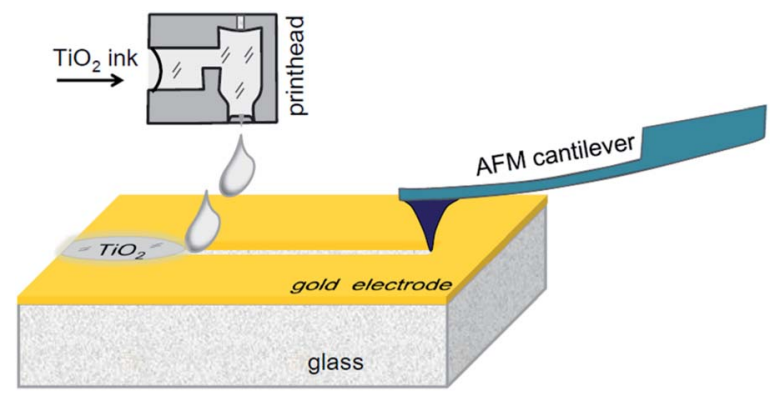

Fig. 1 Scheme for hybrid fabrication of a planar memristor. 


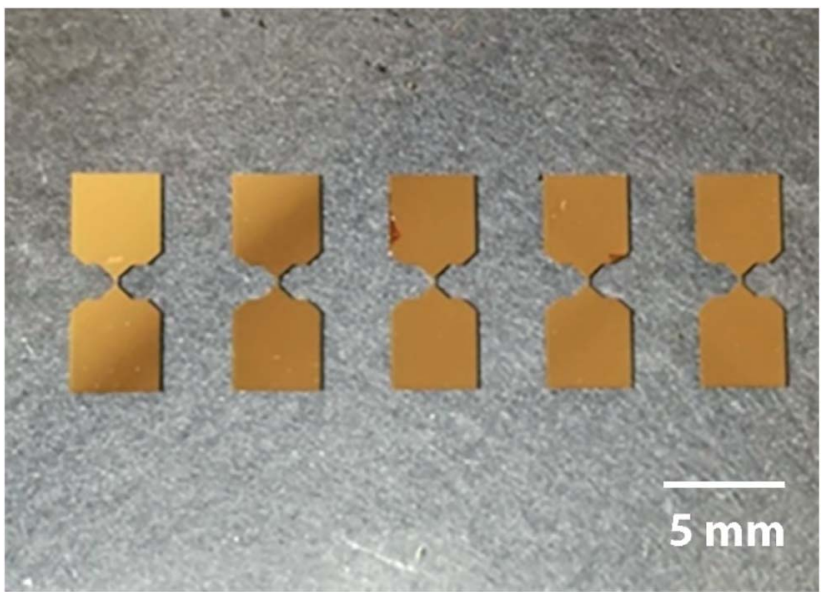

Fig. 2 Gold-sputtered electrodes.

routine $^{37}$ with standard silicon semi-contact probes was performed prior to main operations.

The trenches of the second type were etched by a focused ion beam of Ga using a Carl Zeiss Neon 40 Cross-Beam workstation. Acceleration voltage and current in the beam column were $30 \mathrm{kV}$ and $10 \mathrm{pA}$, correspondingly.

Trench filling. The trenches separating the electrodes were filled with titania $\left(\mathrm{TiO}_{2}\right)$ nanoparticles dispersed in a sol and deposited by inkjet printing. Titania nanoparticles were derived by sol-gel process. The sol was obtained by hydrolysis of titanium(Iv) isopropoxide (TIP) (97\%, Sigma-Aldrich) in deionized water $\left(<5 \mu \mathrm{S} \mathrm{cm}^{-1}\right)$ under vigorous stirring $(1000 \mathrm{rpm})$, while nitric acid (65\%, Sigma-Aldrich) was used for protonating. The whole processing route is described in detail elsewhere. ${ }^{38}$ The resulting colloid was stable, with typical characteristics of zeta potential $c a .20 \mathrm{mV}$ and the average hydrodynamic radius of particles $9.2 \pm 0.9 \mathrm{~nm}$, in agreement with the previous report. ${ }^{39}$

The synthesized sol was processed in a rotary evaporator until the solid $\mathrm{TiO}_{2}$ phase was obtained. Then the ink was derived by diluting $\mathrm{TiO}_{2}$ nanoparticles in deionized water and adding ethylene glycol and a surfactant DX4000 in order to adjust the surface tension and viscosity of the ink solution. The ink rheological properties were adjusted to comply with the technological printing environment (the printhead nozzle diameter and the desirable droplet volume).

The phase composition and crystallinity of the prepared titania ink were characterized by X-ray diffraction and gas sorption methods, using a Rigaku SmartLab 3 diffractometer (CuK $\alpha$ radiation, $\lambda=1.5418 \AA$ ) and a Quantachrome Nova 1200e surface area analyser, respectively. The surface area and the average pore diameters were estimated using the models by Brunauer-EmmettTeller (BET) and Barrett-Joyner-Halenda, (BJH) respectively.

The prepared $\mathrm{TiO}_{2}$ ink was decanted into a cartridge and inkjet deposited onto the trenches by inkjet printing. A Fujifilm Dimatix materials printer DMP- 2831 with a $10 \mathrm{pL}$ cartridge was used. The printing conditions were set as follows: temperature of the substrate $35{ }^{\circ} \mathrm{C}$, nozzle driving voltage $22 \mathrm{mV}$, drop spacing $25 \mu \mathrm{m}$, and $1 \mathrm{~mm}$ sample-to-printhead distance.
Annealing. The prepared films were dried for 1 day at room temperature, then annealed in an air oven at $200^{\circ} \mathrm{C}$ for 2 hours, as suggested in previous studies. ${ }^{40,41}$

\section{Characterization}

The quality of trench forming was controlled by the means of atomic force microscopy (AFM) and scanning electron microscopy (SEM), while the final device prototypes were subjected to electric characterization, as well as to cell viability assay, using the standard methylthiazol tetrazolium (MTT) test protocol.

AFM and SEM imaging. The AFM images were obtained using a scanning probe microscope (SPM) Ntegra Aura (NTMDT, Russia) operating in the AFM contact mode.

An additional visualization of microstructure was obtained using a scanning electron microscope (SEM) Tescan Vega 3 with a beam accelerating voltage of $20 \mathrm{kV}$, operating with a secondary electron detector.

Electrical characterization. Electrical characterization of the planar memristor elements was performed using a SubFemtoamp Remote SourceMeter Keithley 6430 operating in standard two-point regime and KickStart Instrument Control Software Version 2.0.6 (Tektronix). The measurements of electric current were taken by applying a de voltage sweep from 0 to $5 \mathrm{~V}$ stepwise, with a step value of $0.05 \mathrm{~V}$ and a settling time of $0.01 \mathrm{~s}$. All the electrical characterizations were performed at room temperature and normal atmospheric conditions.

Cytotoxicity assay. To assess the cytotoxicity of materials used in the memristor device, a series of samples coated with sputtered $\mathrm{Au}$, as well as samples coated with $\mathrm{TiO}_{2}$ ink were prepared separately and placed into clear 96-well flat-bottom plates (Eppendorf). An additional empty plate was reserved for the control test. The cells of neuroblastoma (IMR-32, ATCC® CCL-127 $7^{\mathrm{TM}}$, USA) and postnatal human fibroblast (PHF, Institute of Cytology RAS, Russia) lines were taken each in a quantity of $5 \times 10^{3}$ and seeded in the prepared 96-well plates with the samples, as well as in the control one. The cell viability was measured using the standard methylthiazol tetrazolium (MTT) assay. ${ }^{42}$ In brief, the cells were incubated for $72 \mathrm{~h}$ at $37^{\circ} \mathrm{C}$. The incubated product was tested by adding $0.2 \mathrm{~mL}$ of MTT $(5 \mathrm{mg}$ $\mathrm{mL}^{-1}$ ) for 2 hours, then the MTT-formazan product was dissolved in $0.2 \mathrm{~mL}$ of DMSO and the optical absorbance was measured at $570 \mathrm{~nm}$ using a plate reader Infinite F50 (Tecan). To improve cells visibility, trypan blue solution (0.4 wt $\%)$ was added as cell stain. The experimental reproducibility was provided with three individual batches prepared for each of the experimental tests.

\section{Results and discussion}

\section{Topology}

Profiling of the trench by an AFM cantilever tip requires adjustment of scratching force and speed towards the optimal regime. In this study we have investigated the relationships between the applied scratching force and the profile of the resulting trench in the gold layer. The results are shown in Fig. 3. Fig. 3a illustrates the AFM images for a series of trenches 
obtained at forces of $2,5,8,12,15$, and $20 \mu \mathrm{N}$, while the corresponding depth profiles are shown in Fig. $3 \mathrm{~b}$. The topology parameters of obtained trenches are listed in Table 1. The results demonstrate that the scratching efficiency changes drastically when the applied force is sufficient to break through the whole layer. In the case of $30 \mathrm{~nm}$ gold film, this was achieved at $20 \mu \mathrm{N}$.

The optimal scratching speed of $3 \mu \mathrm{m} \mathrm{s}^{-1}$ has been found empirically. At a higher speed, scratching was accompanied with a ploughing effect, while speed lowering only resulted in longer processing time.

Fig. 4 compares the trenches obtained by AFM scratching (Fig. 4a) and FIB etching (Fig. 4b). It is noteworthy that the shape of the AFM-processed trench is strongly affected by the geometry of the cantilever tip, so that it is narrow at the bottom (ca. 150-200 $\mathrm{nm}$ ) and broadening towards the top (ca. 700-800 nm). In contrast, the FIB-etched trench has regular rectangularly shaped profile, $c a$. $400 \mathrm{~nm}$ width (Fig. 4b).

\section{Dielectric layer}

Sol-gel synthesis of $\mathrm{TiO}_{2}$ nanoparticles is a low temperature process with a possibility to control the phase ratio of different polymorphs as well as the morphology by adjusting parameters of acidic peptization in an aqueous solution. ${ }^{39}$ In this study we used the regime of synthesis previously adjusted to meet the rheological requirements for inkjet printing. ${ }^{38,43}$ XRD pattern of the derived ink is shown in Fig. 5. The synthesized nanoparticles are predominantly identified as anatase (JSPDS card \#21-1272), while some traces of brookite have also been detected (JSPDS card \#29-1360); no rutile (JSPDS card \#21-1276) is observed. Anatase is the well suitable $\mathrm{TiO}_{2}$ polymorph for memristive application that has been demonstrated in a series of theoretical ${ }^{\mathbf{4 4}}$ and experimental ${ }^{\mathbf{4 5 - 4 7}}$ studies. The average crystallite size by Scherer's equation was $c a .6 \mathrm{~nm}$ upon heat treatment at $50{ }^{\circ} \mathrm{C}$ and $\mathrm{ca} .7 \mathrm{~nm}$ after treatment at $200{ }^{\circ} \mathrm{C}$. The BET

(a)
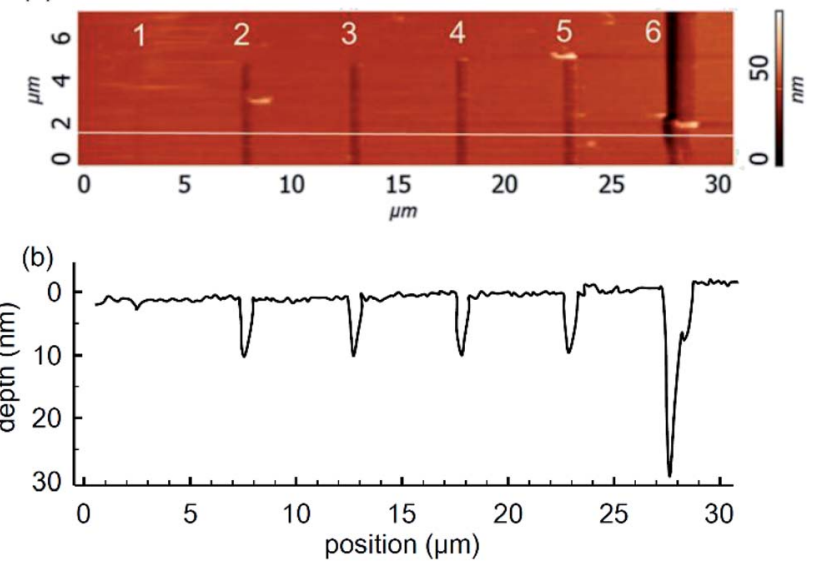

Fig. 3 (a) AFM images of trenches formed with cantilever forces of 2 , $5,8,12,15$, and $20 \mu \mathrm{N}$; and (b) the corresponding depth profiles.
Table 1 Topology of trenches at various forces

\begin{tabular}{lllr}
\hline \multirow{2}{*}{ Scratch number, Fig. 3a } & Force, $\mu \mathrm{N}$ & \multicolumn{2}{l}{ Width at middle, } \\
\hline 1 & 2 & $300 \pm 100$ & Depth, nm \\
2 & 5 & $400 \pm 100$ & $1.5 \pm 0.5$ \\
3 & 8 & $400 \pm 100$ & $8.5 \pm 0.5$ \\
4 & 12 & $500 \pm 100$ & $8.5 \pm 0.5$ \\
5 & 15 & $500 \pm 100$ & $8.5 \pm 0.5$ \\
6 & 20 & $500 \pm 100$ & $30.0 \pm 2.0$
\end{tabular}

surface area was evaluated as $159 \mathrm{~m}^{2} \mathrm{~g}^{-1}$ and $144 \mathrm{~m}^{2} \mathrm{~g}^{-1}$ with the $\mathrm{BJH}$ average pore diameters $3.2 \mathrm{~nm}$ and $3.5 \mathrm{~nm}$ for the samples treated at $50{ }^{\circ} \mathrm{C}$ and $200{ }^{\circ} \mathrm{C}$, respectively.

Fig. 6 demonstrates the result of trench filling by inkjet deposition, followed by annealing. As visible in Fig. 6a, the titania ink droplets are deposited precisely on the trenches. The SEM image shown in Fig. 6b demonstrates the droplet morphology. It forms a compact film with little roughness.

\section{Electric characterization}

The planar $\mathrm{Au} / \mathrm{TiO}_{2} / \mathrm{Au}$ memristors were characterized by current-voltage dependencies at several measurement cycles with stepwise increasing and decreasing driving voltage. The results are shown in Fig. 7. All the memristive elements demonstrated rather very low current response, ranging from a few to hundreds nanoamperes, which may be a result of incomplete or loose filling of the narrow trenches with $\mathrm{TiO}_{2}$ ink and the relatively low crystallinity of titania. Despite this, the U-I dependencies demonstrate the qualitatively memristive behaviour with gradually increasing conductivity at each subsequent unipolar cycle of the applied dc voltage. No electric response
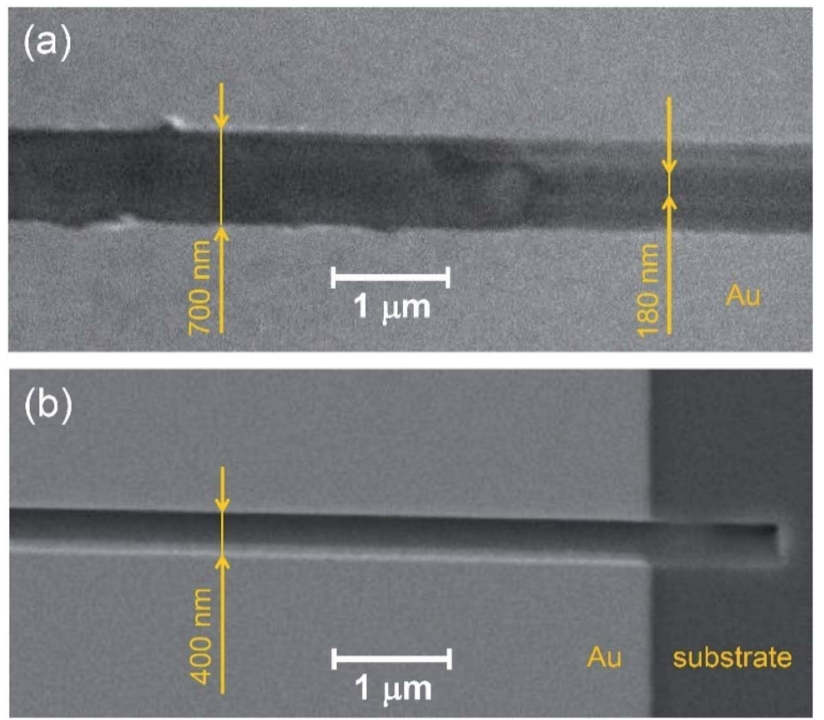

Fig. 4 SEM images of trenches prepared by (a) AFM scratching and (b) FIB etching. 


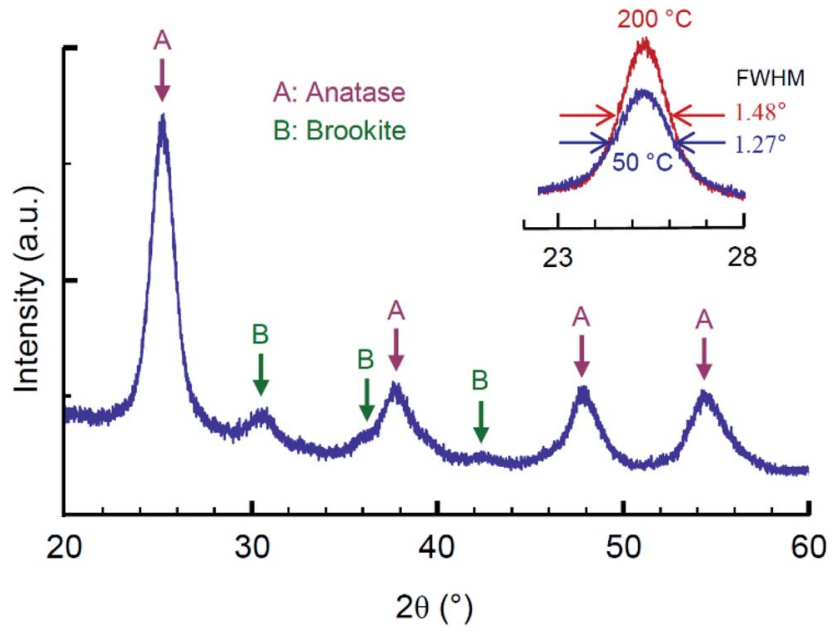

Fig. 5 XRD pattern for sol-gel derived $\mathrm{TiO}_{2}$ ink; the inset shows peaks for materials thermally annealed at $50{ }^{\circ} \mathrm{C}$ and $200{ }^{\circ} \mathrm{C}$.
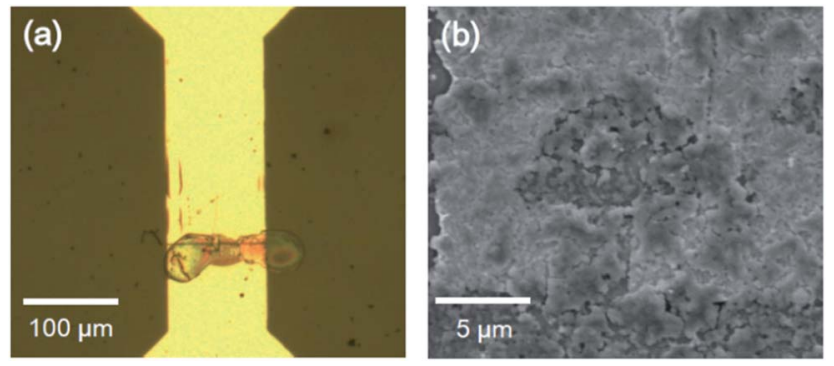

Fig. 6 Images of $\mathrm{TiO}_{2}$ droplets deposited on trench: (a) optical microscopy; and (b) SEM.
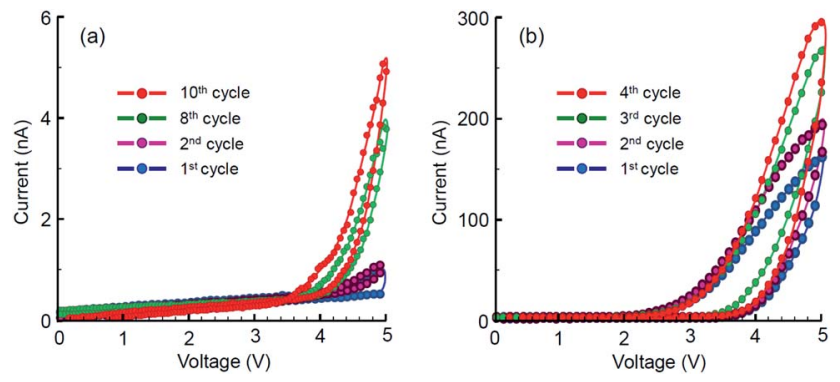

Fig. 7 Current-voltage potentiation curves for a sequence of unipolar cycles applied to memristors prepared with: (a) AFM-scratched trench and (b) FIB-etched trench.

was detected prior to filling the trench with titania ink. The threshold voltages igniting the memristive potentiation were $4.2 \mathrm{~V}$ in the case of AFM-made trench (Fig. 7a) and 3.0 V in the case of FIB-made trench (Fig. 7b), which correspond to the difference in their widths (Fig. $4 \mathrm{a}$ and b). Owing to proper rectangular shape of the FIB-made trench, the threshold potentiation electric field can be calculated and is thus evaluated as $75 \mathrm{kV} \mathrm{cm}^{-1}$.

\section{Biocompatibility}

The fabrication method proposed in this study implies deposition of $\mathrm{Au}$ and $\mathrm{TiO}_{2}$ on the substrate surface. In order to assess biocompatibility of all the materials, they have been deposited separately on a series of glass substrates and subjected to MTT cytotoxicity assays using IMR-32 and PHF cell lines. The experimental results are shown in Fig. 8. The diagram of cell viability is shown in Fig. 8a and demonstrates that none of the materials caused any notable cytotoxic effect on the IMR-32 and PHF cells. Indeed, the cell survival rates for IMR-32 and PHF on $\mathrm{Au}$ and $\mathrm{TiO}_{2}$ were $97.37 \%$ for IMR-32/TiO $2,95.86 \%$ for $\mathrm{PHF} /$ $\mathrm{TiO}_{2}, 95.93 \%$ for IMR-32/Au, and $95.05 \%$ for $\mathrm{PHF} / \mathrm{Au}$ with respect to the control solely incubated batch. In this assay, all the cells were cultivated for 72 hours, the time sufficient for 3 cycles of cell divisions, and no apparent morphological or metabolic changes have been observed, as illustrated in Fig. 8be for all the combinations of the cells and materials addressed in the study. Thus, the memristor elements fabricated by the proposed hybrid planar technology and using $\mathrm{Au}$ and $\mathrm{TiO}_{2}$ as constituting materials have demonstrated high biocompatibility.

\section{Discussion}

The fabrication method addressed in this study has a limited range of applicability, though it has certain favourable advantages for designing in vitro experiments with biological tissues and cells at the lab scale. Indeed, the atomic force microscopy is a well-recognized tool for nanoscale materials patterning and engineering, ${ }^{48,49}$ just as much as for bioengineering, investigations in biochemistry and cell biology. ${ }^{\mathbf{5 0 , 5 1}}$ Inkjet printing is a cost-efficient nanofabrication method, especially for the labscale prototyping. ${ }^{52,53}$ It typically operates with a picolitre droplet volume and 10-20 $\mu \mathrm{m}$ spatial resolution ${ }^{52}$ of the droplet depositing. This is sufficient for integration of the printable microcircuits with biological cells, as long as the cell sizes ( $c a$.
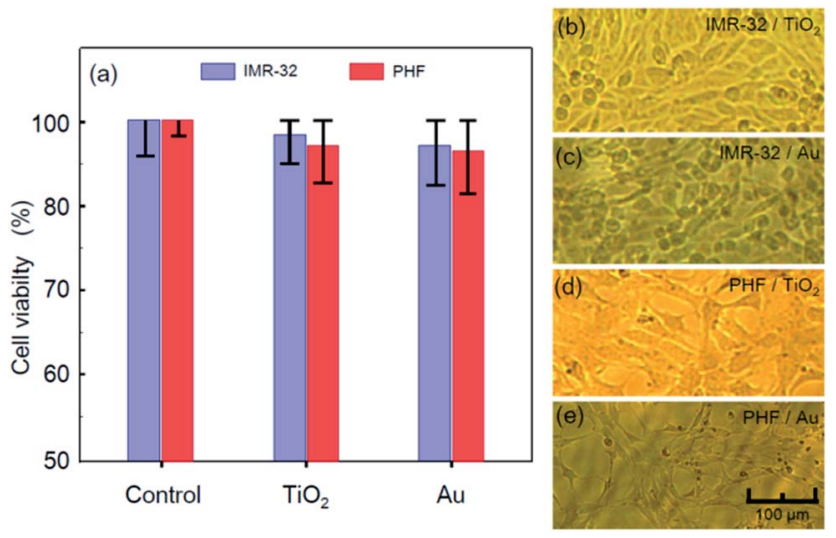

Fig. 8 Viability of IMR-32 and PHF cells incubated solely (control) and on substrates of $\mathrm{TiO}_{2}$ and $\mathrm{Au}$ : (a) chart for percentage of viable cells; and (b-e) optical microscope images of cells after 72 hours of incubation: (b) IMR-32 on $\mathrm{TiO}_{2}$, (c) IMR-32 on $\mathrm{Au}$, (d) $\mathrm{PHF}$ on $\mathrm{TiO}_{2}$, and (e) PHF on Au. 
$100 \mu \mathrm{m}$, e.g. Fig. $8 \mathrm{~d}$ and e) surpass the printing resolution limit. Furthermore, the inkjet bioprinting is rapidly developing nowadays, ${ }^{\mathbf{5 4}, 55}$ thus enabling precise positioning and arrangement of biological and inorganic elements on the substrate.

The nano- and microfabrication methods based on physical $(\mathrm{FIB})^{56}$ or chemical (mask lithography) ${ }^{28,29}$ treatment provide better reproducibility, ${ }^{52}$ though they cannot be considered for conducting in situ experiments with biological cells or tissues. In these cases, the combination of AFM and inkjet patterning shall be a justified choice. Their combination allows for in situ circuit manipulations even in the case with the integrated biological substances.

Seemingly, the major challenge in the proposed design of planar memristors is the relatively low current response experimentally achieved at this stage (Fig. 7a). Nonetheless, we believe that further development of the functional inks shall lead to better performance. Firstly, a more careful adjustment of the ink crystallinity is needed. Technologically, it is feasible for sol-gel ${ }^{38,57}$ and other ${ }^{58}$ processing routes. Secondly, as the concentration of oxygen vacancies in the functional layer significantly affects the memristive performance, ${ }^{44}$ the optimal level of oxygen nonstoichiometry can be achieved by an appropriate doping or by thermal treatment in an inert atmosphere. And thirdly, other biocompatible memristive compositions can be considered besides $\mathrm{TiO}_{2}$.

\section{Conclusion}

In summary, we have designed, fabricated, and investigated a novel planar configuration of a memristive element. The proposed hybrid nanofabrication method allows patterning the insulating trenches in the gold-sputtered electrodes and filling them with functional inks using the AFM and inkjet printing techniques, correspondingly. The combination of these methods allows for operational versatility for designing in vitro experiments with biological cells and tissues. The fabricated planar memristors have demonstrated a memristive behaviour qualitatively, upon U-I characterization. However the electrical response was rather week, possibly due to incomplete filling of the trench or low density of the filling material. Thus the proposed fabrication technique requires further elaboration. Finally, the materials used in the fabrication have demonstrated reasonable biocompatibility, not causing any apparent cytotoxic effect on IMR-32 and PHF cells.

\section{Conflicts of interest}

There are no conflicts to declare.

\section{Acknowledgements}

The research has been carried out with financial support from the Russian Science Foundation (Project No 19-19-00433). AVV acknowledges the scholarship of the President of the Russian Federation (SP-1158.2019.1). The authors acknowledge the Engineering Center at the Saint-Petersburg State Technological
Institute (Technical University) for the support with X-Ray diffraction analysis.

\section{References}

1 L. O. Chua, IEEE Trans. Circuit Theory, 1971, 18, 507-519, DOI: $10.1109 /$ TCT.1971.1083337.

2 L. O. Chua, Appl. Phys. A, 2011, 102, 765-783, DOI: 10.1007/ s00339-011-6264-9.

3 W. Lu, D. S. Jeong, M. Kozicki and R. Waser, MRS Bull., 2012, 37, 124-130, DOI: 10.1557/mrs.2012.5.

4 D. B. Strukov, G. S. Snider, D. R. Stewart and R. S. Williams, Nature, 2008, 453, 80-83, DOI: 10.1038/nature06932.

5 J. J. Yang, F. Miao, M. D. Pickett, D. A. A. Ohlberg, D. R. Stewart, C. N. Lau and R. S. Williams, Nanotechnology, 2009, 20, 215201, DOI: 10.1088/0957-4484/ 20/21/215201.

6 Y. Yang, P. Gao, S. Gaba, T. Chang, X. Pan and W. Lu, Nat. Commun., 2012, 3, 732, DOI: 10.1038/ncomms1737.

7 L. Wang, C. H. Yang, J. Wen, S. Gai and Y. X. Peng, J. Mater. Sci.: Mater. Electron., 2015, 26, 4618-4628, DOI: 10.1007/ s10854-015-2848-z.

8 Q. Xia, W. Wu, G. Y. Jung, S. Pi, P. Lin, Y. Chen, X. Li, Z. Li, S. Y. Wang and R. S. Williams, Appl. Phys. A: Mater. Sci. Process., 2015, 121, 467-479, DOI: 10.1007/s00339-015-9038y.

9 S. Barcelo and Z. Li, Nano Convergence, 2016, 3, 21, DOI: 10.1186/s40580-016-0081-y.

10 Q. Xia, W. Robinett, M. W. Cumbie, N. Banerjee, T. J. Cardinali, J. J. Yang, W. Wu, X. Li, W. M. Tong, D. B. Strukov, G. S. Snider, G. Medeiros-Ribeiro and R. S. Williams, Nano Lett., 2009, 9, 3640-3645, DOI: 10.1021/nl901874j.

11 J. P. Strachan and W. D. Lu, Nat. Electron., 2018, 1, 22-29, DOI: $10.1038 / \mathrm{s} 41928-017-0006-8$.

12 J. J. Yang, D. B. Strukov and D. R. Stewart, Nat. Nanotechnol., 2013, 8, 13-24, DOI: 10.1038/nnano.2012.240.

13 L. O. Chua and S. M. Kang, Proc. IEEE, 1976, 64, 209-223, DOI: 10.1109/PROC.1976.10092.

14 Z. H. Tan, X. B. Yin, R. Yang, S. B. Mi, C. L. Jia and X. Guo, Sci. Rep., 2017, 7, 713, DOI: 10.1038/s41598-017-00849-7.

15 A. L. Hodgkin and A. F. Huxley, J. Physiol., 1952, 117, 500544, DOI: 10.1113/jphysiol.1952.sp004764.

16 C. Li, D. Belkin, Y. Li, P. Yan, M. Hu, N. Ge, H. Jiang, E. Montgomery, P. Lin, Z. Wang, W. Song, J. P. Strachan, M. Barnell, Q. Wu, R. S. Williams, J. J. Yang and Q. Xia, Nat. Commun., 2018, 9, 2385, DOI: 10.1038/s41467-01804484-2.

17 S. Liu, Y. Wang, M. Fardad and P. K. Varshney, IEEE Circuits Syst. Mag., 2018, 18, 29-44, DOI: 10.1109/ MCAS.2017.2785421.

18 B. Widrow, "An adaptive Adaline neuron using chemical memristors”, Stanford Univ., TR, 1960, pp. 1553-2.

19 B. Widrow, ARS J., 1962, 32, 1378-1385, DOI: 10.2514/8.6293.

20 B. Yazmir and M. Reiner, Neuroscience, 2018, 378, 100-112, DOI: $10.1016 /$ j.neuroscience.2016.10.060. 
21 J. J. Daly and J. E. Huggins, Arch. Phys. Med. Rehabil., 2015, 96, S1-S7, DOI: 10.1016/j.apmr.2015.01.007.

22 J. J. S. Norton, J. Mullins, B. E. Alitz and T. Bretl, J. Neural Eng., 2018, 15, 056012, DOI: 10.1088/1741-2552/aacfdd.

23 M. Takemi, T. Maeda, Y. Masakado, H. R. Siebner and J. Ushiba, Neuroimage, 2018, 183, 597-605, DOI: 10.1016/ j.neuroimage.2018.08.070.

24 A. Chiolerio, M. Chiappalone, P. Ariano and S. Bocchini, Front. Neurosci., 2017, 11, 70, DOI: 10.3389/ fnins.2017.00070.

25 P. Massobrio, J. Tessadori, M. Chiappalone and M. Ghirardi, Neural Plast., 2015, 2015, 1-18, DOI: 10.1155/2015/196195.

26 D. Gater, A. Iqbal, J. Davey and E. Gale, in IEEE 20th International Conference on Electronics, Circuits, and Systems (ICECS), IEEE, 2013, pp. 534-537, DOI: 10.1109/ ICECS.2013.6815469.

27 P. J. Kuekes, D. R. Stewart and R. S. Williams, J. Appl. Phys., 2005, 97, 034301, DOI: 10.1063/1.1823026.

28 K. Rajan, S. Bocchini, A. Chiappone, I. Roppolo, D. Perrone, K. Bejtka, C. Ricciardi, C. F. Pirri and A. Chiolerio, Microelectron. Eng., 2017, 168, 27-31, DOI: 10.1016/ j.mee.2016.10.004.

29 K. Rajan, S. Bocchini, A. Chiappone, I. Roppolo, D. Perrone, M. Castellino, K. Bejtka, M. Lorusso, C. Ricciardi, C. F. Pirri and A. Chiolerio, Flexible Printed Electron., 2017, 2, 024002, DOI: $10.1088 / 2058-8585 /$ aa64be.

30 E. J. Sandouk, J. K. Gimzewski and A. Z. Stieg, Sci. Technol. Adv. Mater., 2015, 16, 045004, DOI: 10.1088/1468-6996/16/4/ 045004.

31 Z. Wang, S. Joshi, S. E. Savel'ev, H. Jiang, R. Midya, P. Lin, M. Hu, N. Ge, J. P. Strachan, Z. Li, Q. Wu, M. Barnell, G.-L. Li, H. L. Xin, R. S. Williams, Q. Xia and J. J. Yang, Nat. Mater., 2017, 16, 101-108, DOI: 10.1038/nmat4756.

32 T. Zhang, L. Wang, Q. Chen and C. Chen, Yonsei Med. J., 2014, 55, 283-291, DOI: 10.3349/ymj.2014.55.2.283.

33 C. Minnai, A. Bellacicca, S. A. Brown and P. Milani, Sci. Rep., 2017, 7, 7955, DOI: 10.1038/s41598-017-08244-y.

34 R. Waser and M. Aono, Nat. Mater., 2007, 6, 833-840, DOI: 10.1038/nmat2023.

35 D.-H. Kwon, et al., Nat. Nanotechnol., 2010, 5, 148-153, DOI: 10.1038/nnano.2009.456.

36 A. Wedig, et al., Nat. Nanotechnol., 2016, 11, 67-74, DOI: 10.1038/nnano.2015.221.

37 J. E. Sader, J. W. M. Chon and P. Mulvaney, Rev. Sci. Instrum., 1999, 70, 3967, DOI: 10.1063/1.1150021.

38 A. V. Yakovlev, V. A. Milichko, V. V. Vinogradov and A. V. Vinogradov, ACS Nano, 2016, 10, 3078-3086, DOI: 10.1021/acsnano.5b06074.

39 A. V. Vinogradov and V. V. Vinogradov, J. Am. Ceram. Soc., 2014, 97, 290-294, DOI: 10.1111/jace.12560.
40 N. Samardzic, M. Mionic, B. Dakic, H. Hofmann, S. Dautovic and G. Stojanovic, IEEE Trans. Electron Devices, 2015, 62, 1898-1904, DOI: 10.1109/Ted.2015.2421283.

41 C. Leyva-Porras, A. Toxqui-Teran, O. Vega-Becerra, M. MikiYoshida, M. Rojas-Villalobos, M. García-Guaderrama and J. A. Aguilar-Martínez, J. Alloys Compd., 2015, 647, 627-636, DOI: 10.1016/j.jallcom.2015.06.041.

42 T. Mosmann, J. Immunol. Methods, 1983, 65, 55-63, DOI: 10.1016/0022-1759(83)90303-4.

43 A. V. Yakovlev, V. A. Milichko, V. V. Vinogradov and A. V. Vinogradov, Adv. Funct. Mater., 2015, 25, 7375-7380, DOI: $10.1002 /$ adfm.201503483.

44 A. Paris and S. Taioli, J. Phys. Chem. C, 2016, 120(38), 2204522053, DOI: 10.1021/acs.jpcc.6b07196.

45 V. Prusakova, C. Collini, M. Nardi, R. Tatti, L. Lunelli, L. Vanzetti, L. Lorenzelli, G. Baldi, A. Chiappini, A. Chiasera, D. Ristic, R. Verucchi, M. Bortolottia and S. Dirè, RSC Adv., 2017, 7, 1654, DOI: 10.1039/C6RA25618J.

46 N. Samardzić, M. Mionić, B. Dakić, H. Hofmann, S. Dautović and G. Stojanović, IEEE Trans. Electron Devices, 2015, 62(6), 1898-1904, DOI: 10.1109/TED.2015.2421283.

47 N. Duraisamy, N. Muhammad, H.-C. Kim, J.-D. Jo and K.-H. Choi, Thin Solid Films, 2012, 520, 5070-5074, DOI: 10.1016/j.tsf.2012.03.003.

48 G. Liu, M. Hirtz, H. Fuchs and Z. Zheng, Small, 2019, 1900564, DOI: 10.1002/smll.201900564.

49 X. N. Xie, H. J. Chung, C. H. Sow and A. T. S. Wee, Mater. Sci. Eng., $R$, 2006, 54, 1-48, DOI: 10.1016/j.mser.2006.10.001.

50 J. L. Toca-Herrera, ChemSusChem, 2019, 12, 603, DOI: 10.1002/cssc.201802383.

51 M. Li, N. Xi, Y. Wang and L. Liu, Nano Res., 2019, 12, 703, DOI: $10.1007 / \mathrm{s} 12274-018-2260-0$.

52 E. Menard, M. A. Meitl, Y. Sun, J.-U. Park, D. J.-L. Shir, Y.-S. Nam, S. Jeon and J. A. Rogers, Chem. Rev., 2007, 107, 1117-1160, DOI: 10.1021/cr050139y.

53 L. Nayak, S. Mohanty, S. Nayak and A. Ramadoss, J. Mater. Chem. C, 2019, 7, 8771, DOI: 10.1039/c9tc01630a.

54 A. K. Miri, I. Mirzaee, S. Hassan, S. M. Oskui, D. Nieto, A. Khademhosseini and Y. S. Zhang, Lab Chip, 2019, 19, 2019-2037, DOI: 10.1039/c8lc01037d.

55 R. Zimmermann, C. Hentschel, F. Schrön, D. Moedder, T. Büttner, P. Atallah, T. Wegener, T. Gehring, S. Howitz, U. Freudenberg and C. Werner, Biofabrication, 2019, 11, 045008, DOI: 10.1088/1758-5090/ab2aa1.

56 V. I. Avilov, O. A. Ageev, I. L. Jityaev, A. S. Kolomiytsev and V. A. Smirnov, Proc. SPIE, 2016, 10224, DOI: 10.1117/ 12.2267084.

57 E. Cerro-Prada, S. García-Salgado, M. Á. Quijano and F. Varela, Nanomaterials, 2019, 9, 26, DOI: 10.3390/ nano9010026.

58 M. Zimmermann, B. Temel and G. Garnweitner, Chem. Eng. Process., 2013, 74, 83-89, DOI: 10.1016/j.cep.2013.08.001. 